Document downloaded from:

http://hdl.handle.net/10251/157194

This paper must be cited as:

Ferrando-Rocher, M.; Herranz Herruzo, JI.; Valero-Nogueira, A.; Bernardo Clemente, B. (2019). Full-Metal K-Ka Dual-Band Shared-Aperture Array Antenna Fed by Combined Ridge-Groove Gap Waveguide. IEEE Antennas and Wireless Propagation Letters. 18(7):1463-1467. https://doi.org/10.1109/LAWP.2019.2919928

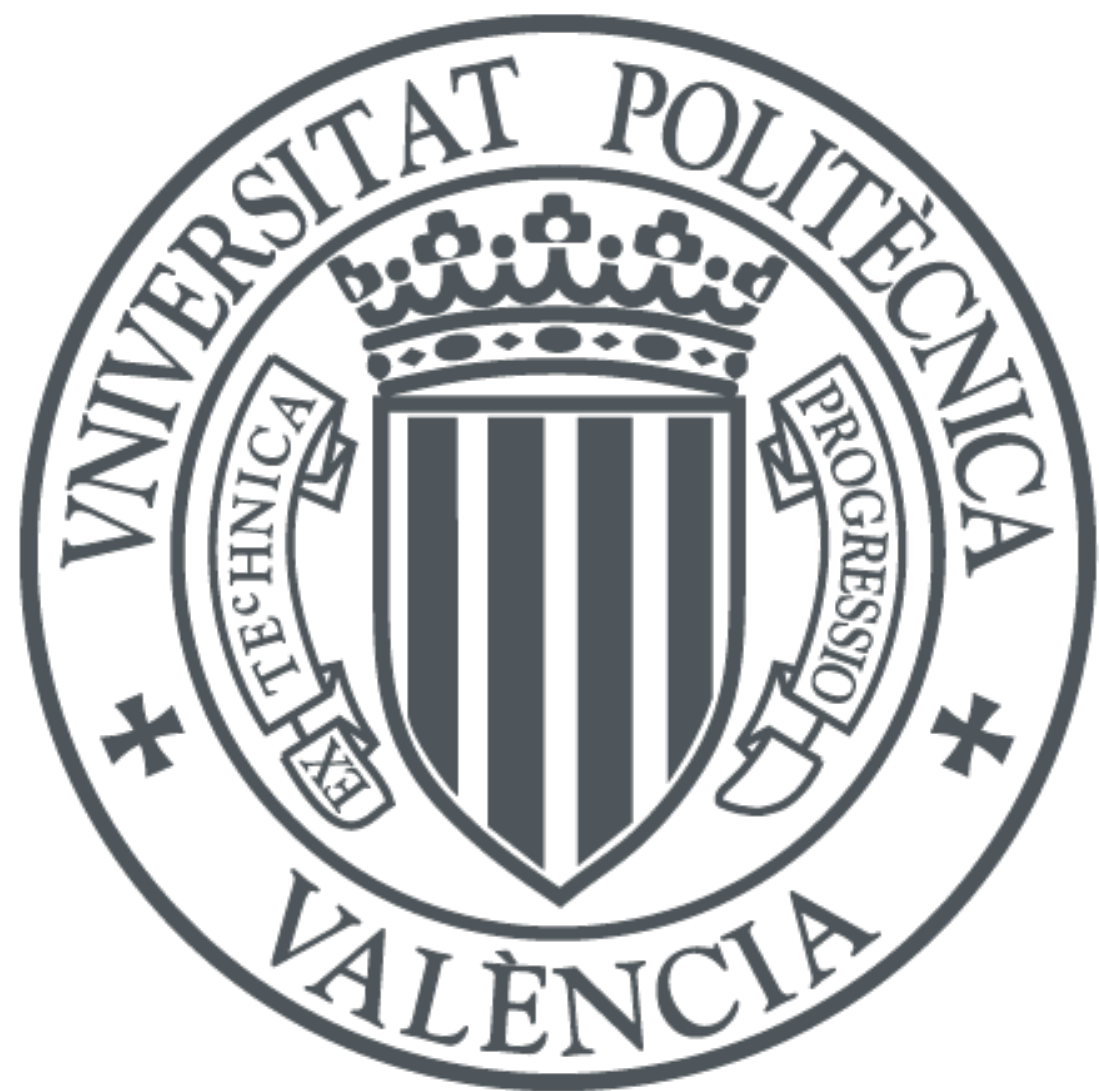

The final publication is available at

https://doi.org/10.1109/LAWP.2019.2919928

Copyright Institute of Electrical and Electronics Engineers

Additional Information

(C) 2019 IEEE. Personal use of this material is permitted. Permissíon from IEEE must be obtained for all other uses, in any current or future media, including reprinting/republishing this material for advertisíng or promotional purposes, creating new collective works, for resale or redistribution to servers or lists, or reuse of any copyrighted component of this work in other works. 


\title{
Full-metal K-Ka Dual-Band Shared-Aperture Array Antenna fed by combined Ridge-Groove Gap Waveguide
}

\author{
Miguel Ferrando-Rocher, Student Member, IEEE, Jose I. Herranz-Herruzo, Member, IEEE, \\ Alejandro Valero-Nogueira, Senior Member, IEEE, Bernardo Bernardo-Clemente
}

\begin{abstract}
This paper presents an $8 \times 8$ dual-band sharedaperture array antenna operating in $\mathrm{K}(19.5-21.5 \mathrm{GHz})$ and $\mathrm{Ka}$ band (29-31 GHz) using Gap Waveguide technology. Radiating elements consist of circular apertures located on the top plate of the antenna and excited by two stacked cavities with different diameters for dual-frequency operation. A waffle grid is used on top to increase the effective area of apertures and reduce grating lobes. Each stacked cavity is fed by its appropriate corporate-feeding network: the upper feeding layer operates at 20-GHz band and the lower one at 30-GHz band. As a result, the antenna presents two ports, one for each band, which radiate a directive farfield pattern with linear polarization, orthogonal to each other. Experimental results show impedance and radiation pattern bandwidths larger than $1.5 \mathrm{GHz}$ in both bands.
\end{abstract}

Index Terms-Dual-Band Antenna, Ka-Band, K-Band, Gap Waveguide, Array, SATCOM, Shared-Aperture, Ridge Gap Waveguide, Groove Gap Waveguide.

\section{INTRODUCTION}

Today, the integration of multiple systems has become one of the development trends in wireless technologies. Therefore, there is growing interest in shared apertures for multi-band, multi-polarized or multi-functional antennas [1]-[4]. They are attractive solutions since the number of antennas on the platform can be reduced and the aperture utilization ratio can be greatly improved. Nonetheless, this type of structures has rarely been addressed beyond the K-band and are usually based on printed solutions. The reduction in the number of antennas is especially appealing in satellite communications where high efficiency is also a must. Therefore, purely metallic structures operating at two distinct non-adjacent frequency bands are highly appreciated.

Mobile satellite terminals need to be lightweight, lowprofile, and capable of tracking satellites. As a consequence of this demand, there is currently a wide interest for innovative antennas able to fullfill all or part of these needs. Planar, lowprofile, lightweight, high-gain, dual-polarized and dual-band array solutions have become a popular research area over the classical solution using parabolic reflectors.

This work was supported by the Spanish Ministry of Economics and competitiveness under projects TEC2016-79700-C2-1-R

M. Ferrando-Rocher, J. I. Herranz-Herruzo, A. Valero-Nogueira, and B. Bernardo-Clemente are with the Instituto de Telecomunicaciones y Aplicaciones Multimedia (ITEAM) of the Universitat Politècnica de València, $\mathrm{c} /$ Cami de Vera s/n, 46022 Valencia, Spain (e-mail: miferroc@iteam.upv.es, jiherhe@upvnet.upv.es, avalero@dcom.upv.es, berbec1@iteam.upv.es)

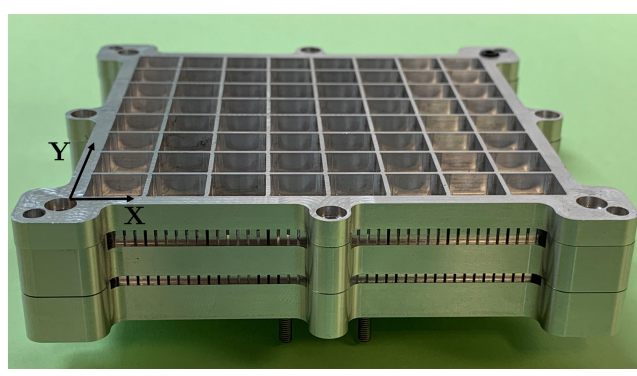

(a)

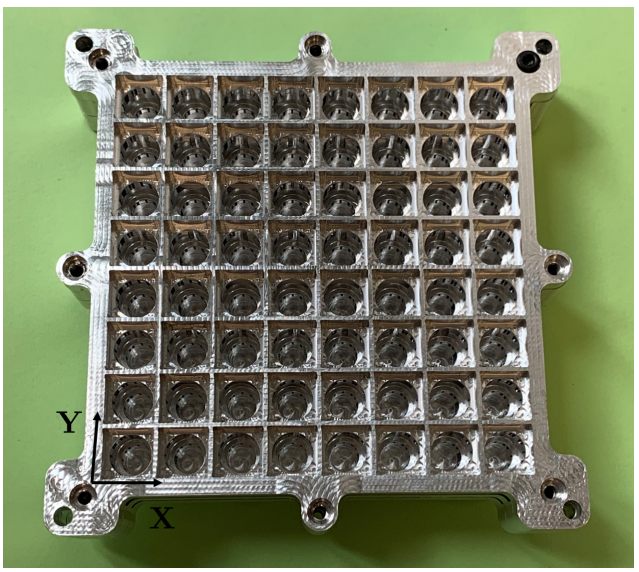

(b)

Fig. 1: Manufactured $8 \times 8$ dual-band array antenna.

In the last decade, gap waveguide (GW) technology has proven to be a suitable candidate to develop high-gain lowprofile antennas in the millimeter-wave band [5]. The fundamental principle of operation of GW relies upon two parallel plates, being one a perfect electric conductor and the other a high-impedance surface, separated less than a quarter wavelength and realized by a textured surface made of periodic metal nails. Then, a groove or a ridge embedded in such surface allows a confined field propagation along the created path. Here, a corporate feeding network combining ridges and grooves is used. This compact network provides a uniform power distribution suitable for array feeding, as successfully demonstrated in other GW array antennas in Kaband [6]. Taking advantage of these GW features, a multitude of linearly-polarized [7], circularly-polarized [8] or dualpolarized antennas [9] have been developed already. However, 


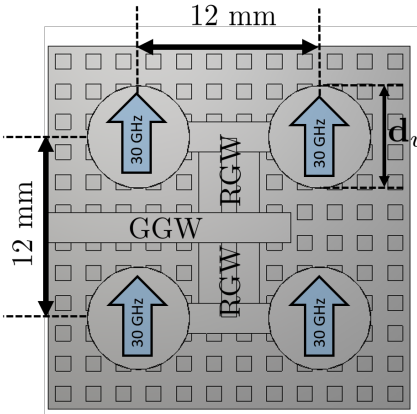

30-GHz Layer

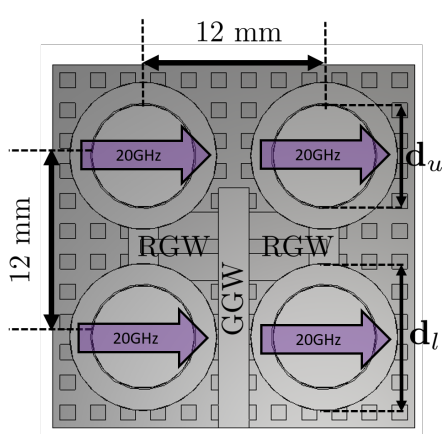

20-GHz Layer
Fig. 2: Top view of the feeding layers for the basic cell $(2 \times 2$ subarray).

the dual-band capability has not been addressed yet.

In this work, we present a full-metal low-profile dual-band shared-aperture array antenna (see Fig. 1) with high gain (above $25 \mathrm{dBi}$ in the lower band and close to $30 \mathrm{dBi}$ in the upper band), high efficiency (above $80 \%$ in both bands), and wide impedance bandwidth ( $\geq 1.5 \mathrm{GHz}$ in both bands too). The proposed array architecture is appropriate for satellite communication terminals, whose targeted bands are in the range of $20-\mathrm{GHz}$ for receive (K-band) and $30-\mathrm{GHz}$ for transmit (Ka-band).

\section{II. $2 \times 2$ DUAL-BAND BASIC CELL}

Figs. 2 and 3 show different views of the $2 \times 2$ dual-band unit cell. Each radiating element consists of a circular aperture, excited by two stacked cylindrical cavities, which in turn are fed from their side. For efficient radiation, the cavity radius must be $\lambda_{0} / \pi$, being $\lambda_{0}$ the free-space wavelength. Taking this fact into consideration, stacked cavities with different diameters are combined for a dual-band operation. Hence, the diameter of the cavity working at $f_{l}=20 \mathrm{GHz}$ is $\mathrm{d}_{l}=9.5 \mathrm{~mm}$ while that operating at $f_{u}=30 \mathrm{GHz}$ is $\mathrm{d}_{u}=6.5 \mathrm{~mm}$. Obviously, the $30-\mathrm{GHz}$ cavity is placed below, so that the field at $30 \mathrm{GHz}$ can propagate through the cylindrical $20-\mathrm{GHz}$ waveguide to finally be radiated. This architecture is inspired by the proposal in [9]. The main difference now is to use cavities with different radius to achieve the dual-band performance. Fig. 3 shows an exploded view of the stacked cylindrical cavities. It is observed that cavities at the two layers are fed at $90^{\circ}$ from each other, hence exciting orthogonal field polarization.

Regarding the textured surface, both layers present some slight differences. The nails of the lower layer have a height of a quarter wavelength at $30 \mathrm{GHz}$ (Ka-band), which is $\mathrm{h}_{u}=2 \mathrm{~mm}$. In the upper layer, where the center frequency is around $20 \mathrm{GHz}$ (K-band), the height of the nails is $\mathrm{h}_{l}=3.5 \mathrm{~mm}$. Nail width $\left(\mathrm{w}_{\text {nail }}=1.4 \mathrm{~mm}\right)$ and periodicity $\left(\mathrm{p}_{\text {nail }}=2 \mathrm{~mm}\right)$ are identical in both layers for ease of design. The distance between apertures is $12 \mathrm{~mm}$, i.e., $0.8 \lambda_{l}$ at $20 \mathrm{GHz}$ and $1.2 \lambda_{u}$ at $30 \mathrm{GHz}$. This separation between apertures would lead to unacceptable grating lobes at $30 \mathrm{GHz}$ but they are mitigated with a waffle-grid plate, as described in [8].
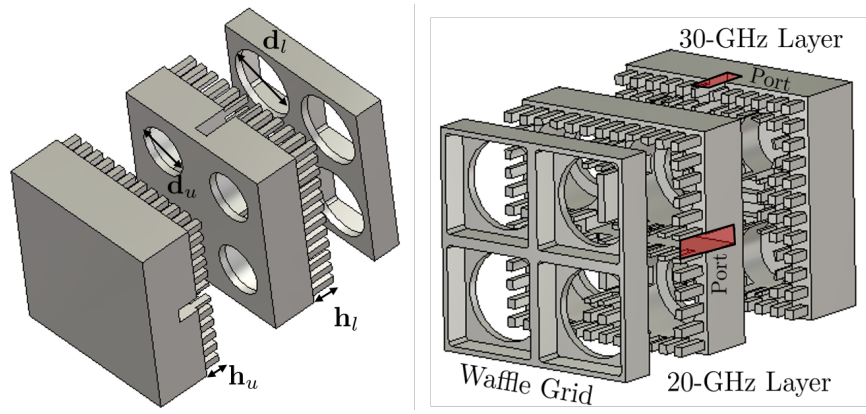

Fig. 3: Layers of the dual-band basic cell.

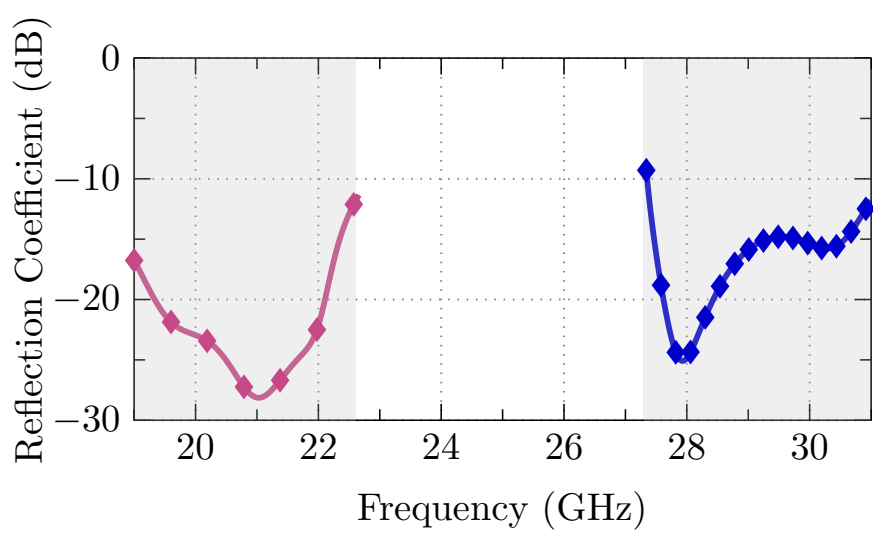

Fig. 4: Simulated reflection coefficient of the $2 \times 2$ basic cell.

\section{A. Simulated results}

The $S_{11}$-parameter for both ports of the $2 \times 2$ basic cell is plotted in Fig. 4. Periodic boundary conditions have been used to emulate a larger array. K-band and Ka-band operation bandwidths are clearly distinguished. The reflection coefficient at the lower band port is below $-20 \mathrm{~dB}$ from $19.5 \mathrm{GHz}$ to $22 \mathrm{GHz}$, covering beyond the bandwidth of interest. $\mathrm{S}_{11^{-}}$parameter at the upper band port is better than $-15 \mathrm{~dB}$ from $27.5 \mathrm{GHz}$ to $30.5 \mathrm{GHz}$, thus obtaining more than $10 \%$ of relative bandwidth. In Fig. 5 simulated normalized radiation patterns in XZ and YZ-planes for both bands are shown, where a $4 \times 4$ array factor has been applied. Predictably, due to the separation of $1.2 \lambda_{u}$ at $30 \mathrm{GHz}$, grating lobes show up in both planes around $\theta=60^{\circ}$. The rest of the patterns agree with a uniform array distribution and look fairly stable in frequency. These results are experimentally confirmed below.

\section{III. $8 \times 8$ DUAL-BAND ARRAY ANTENNA.}

The full array consists of $4 \times 4$ basic cells like the one described above. Radiating elements are fed by two 1 to 64 corporate-feed networks, one at each layer, shown highlighted in Figs. 6a and 6b. The employed architecture, already described in [10], sequentially combines Ridge (RGW) and Groove Gap Waveguides (GGW) to uniformly feed all cavities in phase. Notice also that the $90^{\circ}$ rotation of the layers favours an improved decoupling between input ports.

Figs. $6 \mathrm{a}$ and $6 \mathrm{~b}$ use a color scheme for a better reading. GGW are colored in red and RGW in green. The $30-\mathrm{GHz}$ 

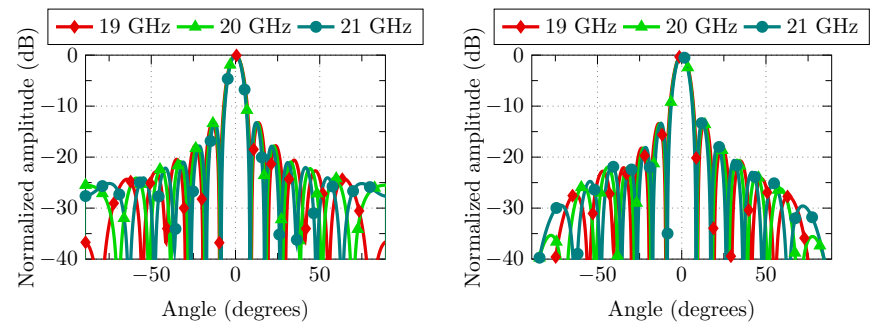

(a)

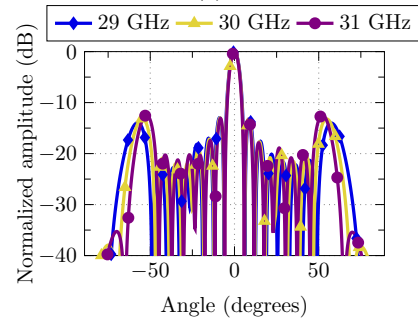

(c)

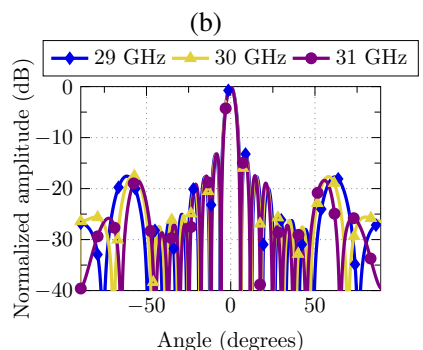

(d)

Fig. 5: Normalized simulated copolar patterns for the basic cell at several frequencies using a $4 \times 4$ array factor: K-band port in (a) XZ-plane and (b) YZ-plane; Ka-band port in (c) XZ-plane and (d) YZ-plane.

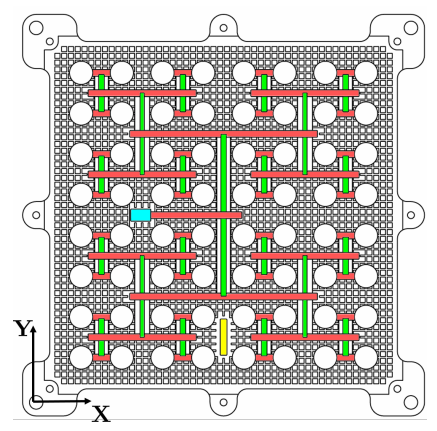

(a)

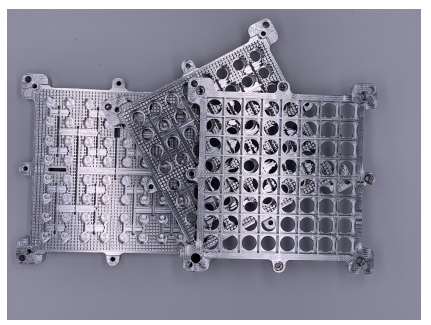

(c)

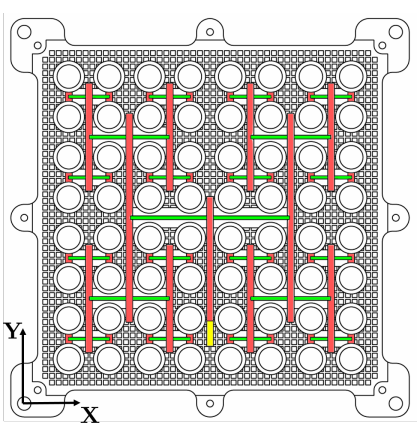

(b)

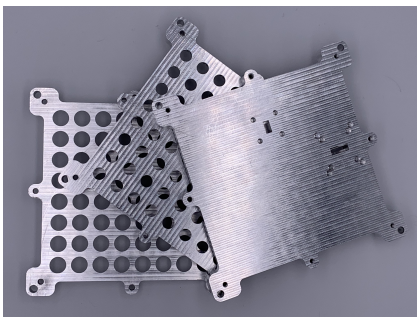

(d)

Fig. 6: (a) Top view of 30-GHz layer; (b) top view of 20-GHz layer. Photograph of the three metallic pieces comprising the manufactured dual-band antenna: (c) top view and (d) bottom view.

input port is colored in blue and the $20-\mathrm{GHz}$ input port in yellow. Note that the $20-\mathrm{GHz}$ port must cross the lower 30 $\mathrm{GHz}$ layer (yellow waveguide in Fig. 6a) to reach the upper layer. The different radius of the cavities in each level can also be observed in these figures. Then, above these two pieces, the top lid is placed. Two pictures of the three aluminum pieces fabricated (top and bottom view) are shown in Figs. 6c and 6d.

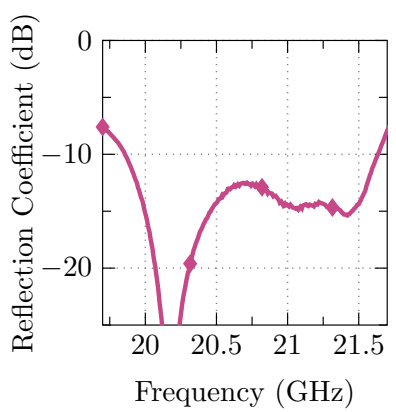

(a)

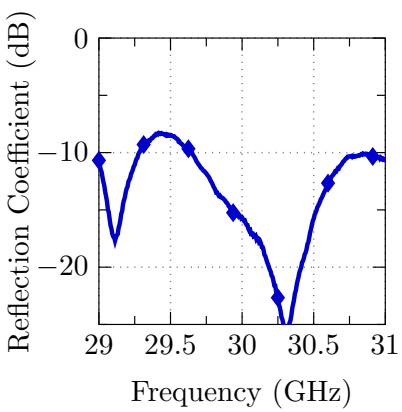

(b)

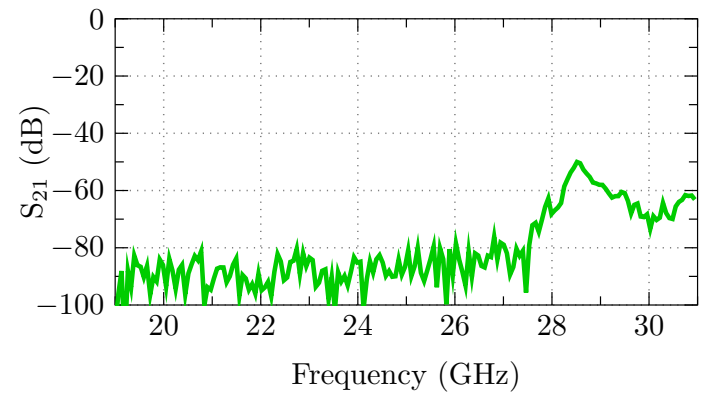

(c)
Fig. 7: Measured S-parameters: (a) $\mathrm{S}_{11}$ at $20-\mathrm{GHz}$ port, (b) $S_{11}$ at $30-\mathrm{GHz}$ port and (c) $S_{21}$.
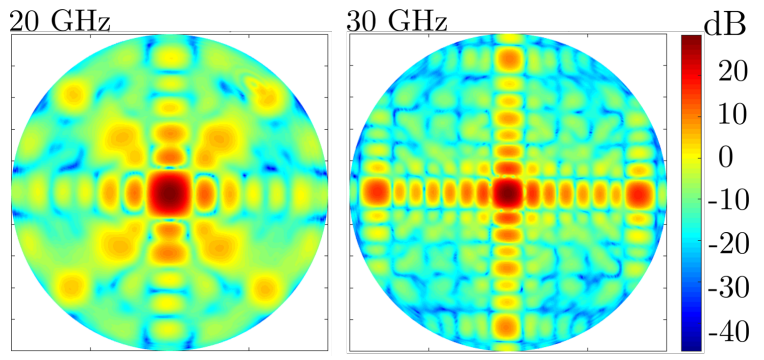

Fig. 8: 2D Measured radiation pattern at $20 \mathrm{GHz}$ (left) and $30 \mathrm{GHz}$ (right).

\section{A. Experimental results}

The experimental results of the manufactured prototype are summarized in Tables I and II and can be observed in Figs. 7, 8 and 9. The first of these figures corresponds to the S-parameters measured for each of the ports.

Reflection coefficient is below $-10 \mathrm{~dB}$ from 19.7 to $21.5 \mathrm{GHz}$, complying broadly with the band of interest (19.7 to $21.2 \mathrm{GHz}$ ). Concerning the upper band, the reflection coefficient is represented from $29 \mathrm{GHz}$ to $31 \mathrm{GHz}$. Even though the $S_{11}$ does not remain below $-10 \mathrm{~dB}$ along the whole represented band, it does so from 29.7 to $31 \mathrm{GHz}$. Thus, the objective of covering the band from $29.5 \mathrm{a} 31 \mathrm{GHz}$ is close to be fulfilled.

With reference to the radiation patterns, 2D representations are displayed in Fig. 8 for each center frequency $(20 \mathrm{GHz}$ and $30 \mathrm{GHz}$ ). Fig. 8 is obtained from an in-house tool that determines the spherical-field modal decomposition. These modes are later used to calculate the far-field at any point [11]. 


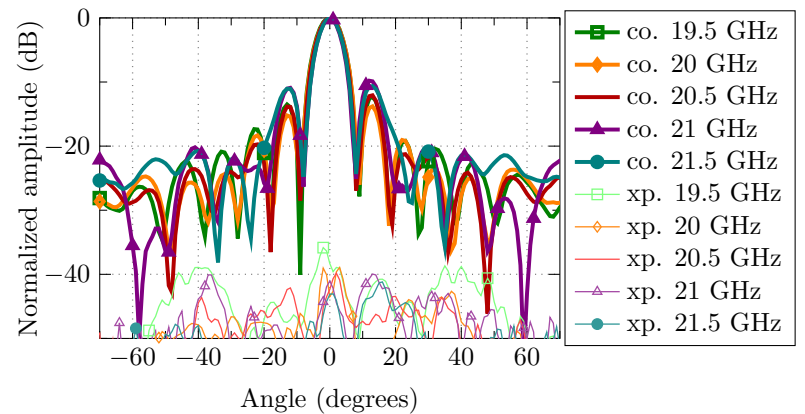

(a)

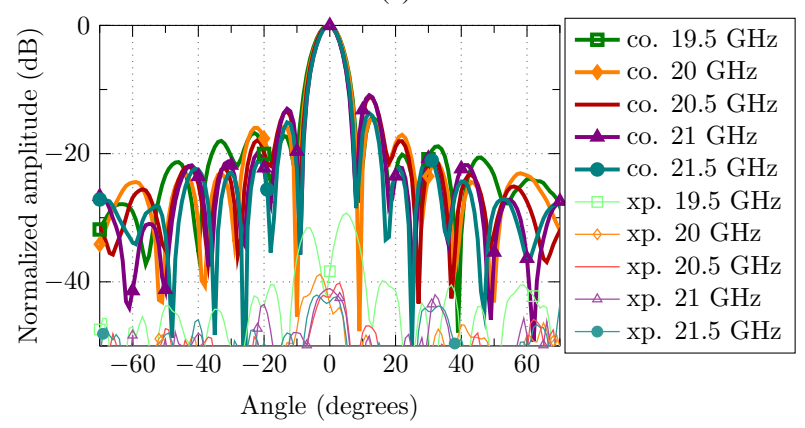

(b)

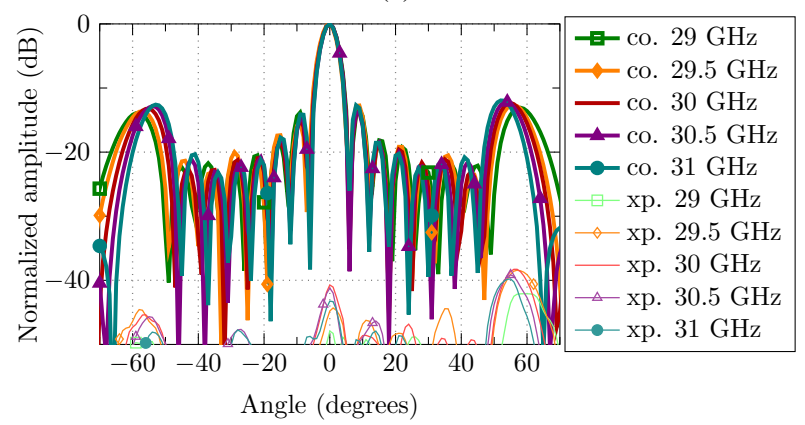

(c)

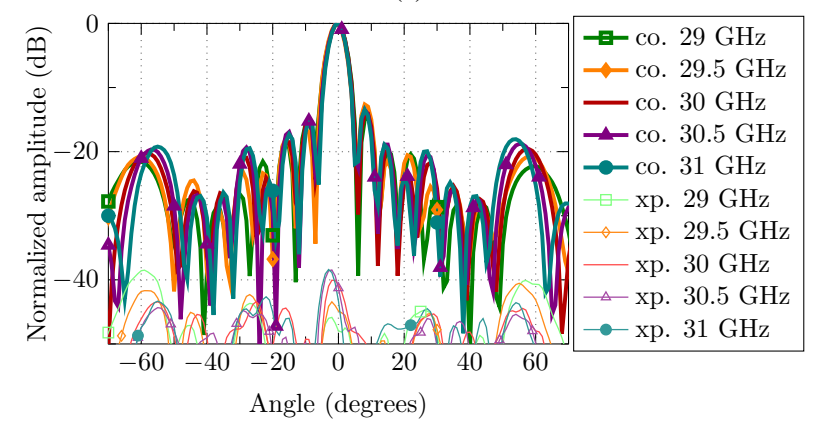

(d)

Fig. 9: Normalized copolar and crosspolar measured radiation patterns for several frequencies: K-band port in (a) XZ-plane and in (b) YZ-plane; Ka-band port in (c) XZ-plane and in (d) YZ-plane.

Besides, in Fig 9 the main cuts are shown at various frequencies in each band: from 19 to $21.5 \mathrm{GHz}$ and from 29 to $31 \mathrm{GHz}$. The results are highly satisfactory, very similar to those obtained in simulation. Grating lobes appearing at the upper band, mostly in the XZ-plane, were predicted by simulations. Their maximum level, however, do not surpass
TABLE I: Measured antenna parameters at K-band.

\begin{tabular}{crrrrc}
\hline Frequency $(\mathrm{GHz})$ & 19.5 & 20.0 & 20.5 & 21.0 & 21.5 \\
\hline \hline Directivity $(\mathrm{dBi})$ & 25.71 & 26.15 & 26.72 & 26.75 & 26.64 \\
\hline Gain $(\mathrm{dBi})$ & 24.68 & 25.40 & 25.86 & 25.85 & 26.03 \\
\hline Antenna Efficiency (\%) & 78.88 & 84.14 & 82.04 & 81.28 & 86.90 \\
\hline
\end{tabular}

TABLE II: Measured antenna parameters at Ka-band.

\begin{tabular}{cccccc}
\hline Frequency $(\mathrm{GHz})$ & 29.0 & 29.5 & 30.0 & 30.5 & 31.0 \\
\hline \hline Directivity $(\mathrm{dBi})$ & 29.55 & 29.56 & 29.83 & 29.87 & 29.98 \\
\hline Gain $(\mathrm{dBi})$ & 28.48 & 29.00 & 29.30 & 29.04 & 29.49 \\
\hline Antenna Efficiency $(\%)$ & 78.15 & 88.03 & 88.44 & 82.58 & 89.42 \\
\hline
\end{tabular}

TABLE III: Performance comparison between proposed and reported dual-band array antennas.

\begin{tabular}{ccccc}
\hline Reference & This Work & {$[4]$} & {$[12]$} & {$[13]$} \\
\hline \hline Bands & K-Ka & K-Ka & L-Ka & Ku-Ka \\
\hline BW (\%) & $10-6$ & n/a & $4-3$ & $16-5$ \\
\hline Max. Gain (dBi) & $26-29.5$ & $24-27$ & $9-29$ & $28-28$ \\
\hline Mean Eff. (\%) & $83-85$ & n/a & n/a-60 & n/a \\
\hline Technology & GW & Transmitarray & $\mu$ strip & Reflectarray \\
\hline Full Metal & Yes & No & No & No
\end{tabular}

the secondary lobe level and may be considered as acceptable.

The measurement data displayed in Tables I and II reveal an antenna efficiency above $80 \%$ in both bands, being the maximum gain $26 \mathrm{dBi}$ and $29.5 \mathrm{dBi}$ in the lower and upper bands, respectively.

Finally, Table III shows a performance comparison between proposed and reported dual-band antennas implemented with different technologies.

\section{CONCLUSIONS}

An $8 \times 8$ dual-band antenna array using Gap Waveguide technology, mainly intended for satellite communications, is presented. The radiating elements consist of shared circular apertures excited by stacked cylindrical cavities, fed in turn by two feeding layers operating at $\mathrm{K}$ and Ka-band. This proof of concept proposes for the first time a full-metal GW antenna with two non-adjacent working bands. Experimental results demonstrate that the proposed scalable array architecture provides the desired dual-band performance as well as a high efficiency and good radiation and impedance bandwidth.

Grating lobes presence due to the larger array spacing at the upper band might be mitigated by several approaches, e.g. by using a frequency selective surface on a superstrate. It should be also noted that the grating lobes level would certainly decrease for other FDD systems with closer bands. Both this issue and the fact of achieving circular polarization are not minor and should be faced to comply with the strict system requirements.

\section{ACKNOWLEDGEMENTS}

This work has been supported by the Spanish Ministry of Economy and Competitiveness (Ministerio de Economía y Competitividad) under the project TEC2016-79700-C2-1-R. 


\section{REFERENCES}

[1] Y. R. Ding and Y. J. Cheng, "Ku/ka dual-band dual-polarized sharedaperture beam scanning antenna array with high isolation," IEEE Transactions on Antennas and Propagation, 2019.

[2] J. F. Zhang, Y. J. Cheng, Y. R. Ding, and C. X. Bai, "A dual-band shared-aperture antenna with large frequency ratio, high aperture reuse efficiency and high channel isolation," IEEE Transactions on Antennas and Propagation, 2018.

[3] F. Qin, S. S. Gao, Q. Luo, C.-X. Mao, C. Gu, G. Wei, J. Xu, J. Li, C. Wu, K. Zheng et al., "A simple low-cost shared-aperture dual-band dual-polarized high-gain antenna for synthetic aperture radars," IEEE Transactions on Antennas and Propagation, vol. 64, no. 7, pp. 29142922, 2016.

[4] S. A. Matos, E. B. Lima, J. S. Silva, J. R. Costa, C. A. Fernandes, N. J. Fonseca, and J. R. Mosig, "High gain dual-band beam-steering transmit array for satcom terminals at ka-band," IEEE Transactions on Antennas and Propagation, vol. 65, no. 7, pp. 3528-3539, 2017.

[5] P.-S. Kildal, E. Alfonso, A. Valero-Nogueira, and E. Rajo-Iglesias, "Local metamaterial-based waveguides in gaps between parallel metal plates," IEEE Antennas and Wireless Propagation Letters, vol. 8, pp. 84-87, 2009.

[6] M. Ferrando-Rocher, J. I. Herranz-Herruzo, A. Valero-Nogueira, and B. Bernardo-Clemente, "Performance assessment of gap-waveguide array antennas: Cnc milling versus three-dimensional printing," IEEE Antennas and Wireless Propagation Letters, vol. 17, no. 11, pp. 20562060, 2018.

[7] A. J. Sáez, A. Valero-Nogueira, J. I. Herranz, and B. Bernardo, "Singlelayer cavity-backed slot array fed by groove gap waveguide," IEEE Antennas and Wireless Propagation Letters, vol. 15, pp. 1402-1405, 2016.

[8] M. Ferrando-Rocher, J. I. Herranz-Herruzo, A. Valero-Nogueira, and A. Vila-Jiménez, "Single-layer circularly-polarized $k a$-band antenna using gap waveguide technology," IEEE Transactions on Antennas and Propagation, vol. 66, no. 8, pp. 3837-3845, 2018.

[9] M. Ferrando-Rocher, J. I. Herranz-Herruzo, A. Valero-Nogueira, B. Bernardo-Clemente, A. Zaman, and J. Yang, " $8 \times 8$ ka-band dualpolarized array antenna based on gap waveguide technology," Accepted for publication: IEEE Transactions on Antennas and Propagation, vol. 67, no. 7, pp. 1-10, 2019.

[10] M. Ferrando-Rocher, A. Valero-Nogueira, and J. I. Herranz-Herruzo, "New feeding network topologies for high-gain single-layer slot array antennas using gap waveguide concept," in Antennas and Propagation (EUCAP), 2017 11th European Conference on. IEEE, 2017, pp. 16541657.

[11] J. Hald and F. Jensen, Spherical near-field antenna measurements. Iet, 1988, vol. 26.

[12] I. Russo, C. Canestri, A. Manna, G. Mazzi, and A. Tafuto, "Dual-band antenna array with superdirective elements for short-distance ballistic tracking," IEEE Transactions on Antennas and Propagation, vol. 67, no. 1, pp. 232-241, 2019.

[13] E. Martinez-de Rioja, J. A. Encinar, M. Barba, R. Florencio, R. R. Boix, and V. Losada, "Dual polarized reflectarray transmit antenna for operation in ku-and ka-bands with independent feeds," IEEE Transactions on Antennas and Propagation, vol. 65, no. 6, pp. 3241-3246, 2017. 